\title{
A Before-After Study of Generic Contextual Diagnostic Labelling and Immediate Therapeutic Interventions Concept Maps for Decision Making
}

\author{
Imad Salah Hassan, Mohammed Al Somaili, Ali Al Khathami, Mohammed Al Ghobain, Salih \\ Bin Salih
}

Department of Medicine, King Saud bin Abdulaziz University for Health Sciences and King Abdulaziz Medical City, Riyadh, Saudi Arabia.

$\begin{array}{ll}\text { ARTICLE INFO } \\ \text { Received } & : 30 / 09 / 2014 \\ \text { Accepted } & : 07 / 01 / 2015 \\ \text { Published } & : 25 / 03 / 2015\end{array}$

KEYWORD

Concept maps

Decision-making

Knowledge Translation

\section{ABSTRACT}

Objective: Cognitive interventions using concept maps to enhance analytical thinking and decision making may improve evidence implementation. Method: The two generic maps that were tested are the BESD diagnosis (Bedside Clinical Diagnosis, Etiological Cause/Precipitant and Severity Score Diagnostic Labeling) and the 5S (Symptomatic, Supportive, Specific, Specialty Referral and Site of Care) concept maps. Trainees in residency training programs were presented with classic case scenarios. They were requested to outline in an answer sheet their diagnosis and their immediate therapeutic interventions. Once completed, the same case scenario was resubmitted to the trainee but this time, the answer sheet was restructured along the two concept maps. The two answer sheets were then compared with regards the explicit inclusion of the various domains of diagnosis and management as outlined in the two maps. Results: A total of 72 trainees were tested. These were grouped into R1 residents and Interns (Juniors, 29 candidates) and R2, R3 and R4 residents (Seniors, 43 candidates). Etiologic labeling improved by $82.8 \%$ in juniors and by $60.5 \%$ in seniors, and severity categorization by $79.3 \%$ and $88.4 \%$ respectively. Symptomatic care inputs increased by $69 \%$ in juniors and $67.1 \%$ in seniors, and documentation of sites of care by $86.2 \%$ and $76.7 \%$ respectively. An extra $75.9 \%$ of juniors and $58.1 \%$ of seniors included specialty referral as part of their immediate therapeutic interventions. Conclusions: The BESD and 5S generic concept maps improved trainees' diagnostic labeling and management decision-making process. Routine use of these maps in residents' training may result in better knowledge translation.

(C) Medical Education Department, School of Medical Sciences, Universiti Sains Malaysia. All rights reserved.

CORRESPONDING AUTHOR: Dr Imad Salah Ahmed Hassan, Assistant Professor/Consultant Physician, Chairman, Knowledge Translation Committee, Department of Medicine 1443, King Saud bin Abdulaziz University for Health Sciences and King Abdulaziz Medical City, P. O. Box 22490, Riyadh 11426, Saudi Arabia. Email: imadsahassan@yahoo.co.uk

\section{Introduction}

Knowledge Translation is "the effective and timely incorporation of evidence-based information into the practices of health professionals in such a way as to affect optimal health care outcomes and maximize the potential of the health system". Successful Knowledge Translation is thus the goal for all healthcare providers. The Institute of Medicine has emphasized that the desired health outcomes must include the following quality of care domains: Patient Safety, Patient-Centeredness, Effectiveness, Efficiency, Timeliness and Equity 
[1]. The knowledge-to Practice gap remains wide despite the enormous strides made in knowledge creation and the availability of numerous effective implementation tools [2]. Cognitive interventions to affect the above goals need to be included as an essential tool for successful implementation of evidence [3].

Several theories of learning have laid the foundation for developing specific and robust teaching curricula that facilitated knowledge acquisition and decision-making [4]. As opposed to the Cognitive Theory of learning which primarily implies a unidirectional teacher to learner knowledge flow, the Constructivist Theory implies a multi-directional and multicomponent knowledge acquisition and assimilation by the learner based on an interaction between the newly acquired knowledge and previous knowledge and experience. The learner "in an active learning mode" gradually develops mental models and schemes for problem solving, decision-making etc [4]. As such, learners will learn best by individually trying to make sense of this 'old and new knowledge and experience' on their own with the teacher acting as a guide to help them develop or "construct"new ideas and concepts along the way [4].

As per the Cognitive Load Theory, an important requirement in optimizing the ability of trainees to learn a new knowledge is to avoid high cognitive loads be it in its thinking framework, complexity or the way it is presented [5]. Additionally, Knowles Androgogy or Adult Learning Theory entails that orientation to learning i.e. "this new knowledge is useful for my immediate needs' is an important motivator for adults to "selectively" acquire new knowledge [6]. The Theory also entails that adults learn better when they see the link between what they learn and their own previous experiences; when they know the objectives of their learning, and when they are actively involved in, and given responsibility for, their learning.

Unlike novices, experts through "experience" acquire mental maps or schemes so called "disease scripts" that allows them to quickly retrieve needed knowledge (by mostly a subconscious recall of pertinent or relevant information) and seamlessly make appropriate and confident decisions. Cognitive individual interventions e.g. through training, mnemonics etc as well as system-based interventions e.g. through clinical pathways or algorithms' etc have been used to specifically aid and improve novices and trainees capacity in their critical thinking and decision-making processes [7,8].

In this study, we aimed at testing two simple concept maps that we believed would significantly enhance trainees' practical, evidence-based, critical thinking and decisionmaking in the diagnostic and therapeutic interventions domains. Concept maps are visual graphic representations of interlinked or interrelated ideas or concepts [9,10]. As cognitive aids, they are useful in creating a conceptual framework that enables users to strategically make important clinical decisions $[10,11]$. In the medical literature, we were unable to identify any generic concept maps or cognitive aids that would enhance a trainee's comprehensive diagnostic labelling or immediate therapeutic interventions. We anticipate that the routine use of these generic models as tools for guiding cognitive decision-making would help realize some of the goals of the Knowledge Translation science.

\section{Method}

The two concept maps namely the BESD and $5 \mathrm{~S}$ models have been described previously [12]. The BESD diagnostic concept map (the Bed-side Clinical Diagnosis, the Etiological or Precipitating Cause and the Severity Score or Grade) is portrayed as a robust model for a more comprehensive, knowledge translation-friendly, diagnostic labeling. For example, failure to consider the precipitant or cause in a patient with a clinical diagnosis of exacerbation of bronchial asthma (e.g. poor inhaler technique) or worsening heart failure (e.g. secondary to pneumonia) will inevitably result in a deficient care input, a poorer outcome and possibly increased future reuse of healthcare resources. 
Appropriate evidence-based interventions to optimize outcome according to severity will be different specifically with regards the sites of care and recommended immediate interventions. In one of the two clinical examples quoted above and according to guidelines recommendations, a mild attack of bronchial asthma entails sites of care (disposition) and emergency therapeutic interventions that are fundamentally different [8].

Similarly, the 5S therapeutic concept map (Site of Care, Symptomatic treatment, Supportive care, Specific Care, Specialty Referral) is considered a simple cognitive aid that will assist the practicing physician (especially front-line staff in the emergency room) in constructing an evidence-based, patient-centered, timely and comprehensive therapeutic plan.

- Site of Care: Guidelines, unambiguously dictate sites of care for specific disease severity scores or categories e.g. in a patient with diabetic ketoacidosis and significant hypokalemia or hyperosmolarity [14].

- Symptomatic treatment: is important as it directly alleviates patient discomfort. Symptom relief is regrettably not regularly ordered by medical staff. An excellent example is the use of analgesics in the acute care setting-so called oligoanalgesia [15].

- Supportive care: to improve physiological derangements before damage becomes irreversible and until the precipitant is brought under control by its specific intervention may be life-saving e.g. oxygen therapy in hypoxic patients, intravenous fluids in patients with hypovolemic shock, sodium bicarbonate in severely acidotic patients etc.

- Specific Care: directed at the primary cause or etiology.

- Specialty Referral: guidelines recommend early specialty or sub-specialty referral for specific acute illnesses e.g. patients with the acute coronary syndromes or significant upper gastrointestinal hemorrhage and associated co-morbidities need to be referred early to their respective specialties.
The two concept maps are depicted in Figure 1. We investigated the use of the above two concept maps namely the BESD model for diagnostic labeling and the $5 \mathrm{~S}$ model for immediate therapeutic decision making.

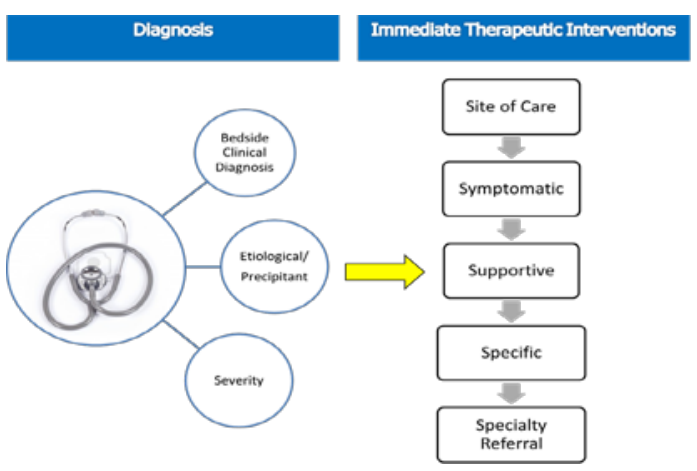

Figure 1: The BESD and 5S Concept Maps

\section{Result}

A total of 43 senior trainees and 29 juniors agreed to take part. 29 residents were from Medicine, 12 from Surgery, 15 from Pediatrics and 16 from Obstetrics and Gynecology. As the number of tested trainees in some specialties is relatively small, a composite pre- and postpercentage inclusion of diagnostic labeling and immediate therapeutic interventions for all juniors and seniors is depicted on Table 2 and shown graphically in Figure 2 and 3.

All domains of the maps showed improvement post-introduction of the two concepts for both the juniors and seniors. These were statistically significant in the inputs regarding etiologic labeling, severity assessment/categorization, symptomatic interventions, selection of the site of care and appropriate specialty referral. Etiologic labeling improved by $82.8 \%$ in juniors and by $60.5 \%$ in seniors. The corresponding increases in severity categorization were $79.3 \%$ and $88.4 \%$ respectively. Similarly, symptomatic care inputs increased by $69 \%$ in juniors and $67.1 \%$ in seniors. More trainees explicitly documented sites of care for each case scenario with an improvement of $86.2 \%$ for juniors and $76.7 \%$ for seniors. Specialty referral was also enhanced with an extra $75.9 \%$ of juniors and $58.1 \%$ of seniors including it as part of their 
immediate therapeutic interventions (Table 2 and Figure 2 and 3).

\section{Table 1: Case Scenarios}

\begin{abstract}
Internal Medicine Clinical Scenario:

\section{Medical Clinical Scenario:}

A 65 year old woman presented to the emergency department complaining of fatigability, epigastric abdominal pain and vomiting. The history is obtained from her daughter as she felt very weak. She had a history of a peptic ulcer for several years for which she has been treated with an $\mathrm{H} 2$ blocker. She also had a history of left knee osteoarthritis of 3 years duration on regular diclofenac tablets for pain relief. According to her daughter, vomitus was blackish in color initially but lately has become reddish in color. She admitted to occasionally passing painless black sticky stool in the last 2 weeks. On examination, patient was drowsy and extremities felt cold. The pulse was $130 \mathrm{bpm}$ and weakly felt, blood pressure 92/61 mmHg. The abdominal examination was remarkable for moderate epigastric tenderness and slight guarding with no rebound tenderness. Rectal examination produced dark stool that was guaiac positive.
\end{abstract}

Answers model:

* What is your bedside clinical diagnosis (the diagnosis that would explain all the symptoms and signs ): Hypovolemic shock secondary to upper gastrointestinal bleeding

* What is your likeliest bedside etiologic etiopathological cause of above bedside clinical diagnosis: Use of non-steroidal anti-inflammatory drug (Diclofenac).

* What is your bedside severity score: Severe

List at least 5 essential immediate therapeutic interventions:

- Site of care: Critical care

- Symptomatic intervention: Analgesics and antiemetics

- Supportive intervention: Intravenous fluids, oxygen, blood transfusion

- Specific intervention: Endoscopy, intravenous proton pump inhibitor.

- Specialty referral: Intensivist, Gastroenterologist

General Surgery Scenario:

An 81 year old man attended ER complaining of severe colicky abdominal pain, distension, nausea and persistent vomiting. He has not passed any gas or stools for the past 12 hours. His pulse was 110/min and blood pressure 92/50. Abdominal examination revealed a distended, tympanic abdomen with hyperactive, highly pitched bowel sounds. There were no signs of peritoneal irritation. Rectal exam was negative for blood or masses and the rectal vault was empty. Abdominal x-ray film showed distended loops of small and large bowel as well as a very large, round, gas shadow that was located in the right upper quadrant tapering towards the left lower quadrant in the shape of parrot beak. There were multiple fluid levels. The patient had a history of abdominal surgery 7 months earlier. There were no palpable hernias.
* What is your bedside clinical diagnosis ( the diagnosis that would explain all the symptoms and signs ): Hypovolemic shock secondary to bowel obstruction

* What is your likeliest bedside etiologic etiopathological cause of above bedside clinical diagnosis: Adhesions from previous abdominal surgery

* What is your bedside severity score: severe

List at least 5 essential immediate therapeutic interventions:

- Site of care: Adult care, ? Critical Care

- Symptomatic intervention: Analgesics, antiemetics and nasogastric suctioning

- Supportive intervention: Intravenous fluids

- Specific intervention: Elective surgical exploration for adhesions

- Specialty referral: General Surgery

\section{Pediatric Case Scenario:}

An 18 month old male is brought to the emergency department with a chief complaint of diarrhea and vomiting for 2 days duration. His mother describes stools as liquid, with no mucous, slime or blood. He reportedly is unable to keep anything down, vomiting after every feeding, even water. His mother reports that he is not feeding well and his activity level is decreased. He has a decreased number of wet diapers. His last weight at his 15 months check up was 25 pounds (11.4 kg).

Exam: VS T 37.8 , P 140, RR 25, BP 78/60, weight 11.3 $\mathrm{kg}$ (40th percentile). He is drowsy, in mother's arms, crying at times, and looks tired. HEENT: anterior fontanel closed, minimal tears, dry lips and mucous membranes. His abdomen is flat, soft, and non-tender with hyperactive bowel sounds. His diaper is dry. His capillary refill time is more than 3 seconds and his skin turgor is diminished. Systemic examination otherwise unremarkable. Initial investigation showed leukocytosis WBC : 17,500 and $\mathrm{Hb} 90 \mathrm{~g} / \mathrm{L}$, Na : 151 (135-145) k: 3.2 (3.5-5.5) HCO3: 14 (18-22) $\mathrm{mEq} / \mathrm{L}$. Stool culture was pending.

* What is your bedside clinical diagnosis ( the diagnosis that would explain all the symptoms and signs ): Hypovolemic shock with hypernatremic dehydration

* What is your likeliest bedside etiologic etiopathological cause of above bedside clinical diagnosis: Viral gastroenteritis

* What is your bedside severity score: Severe

List at least 5 essential immediate therapeutic interventions:

- Site of care: Critical Care

- Symptomatic intervention: Anti-emetics

- Supportive intervention: Keep NPO

- Specific intervention: intravenous hydration

- $\quad$ Specialty referral: Intensivist

\section{Obstetric \& Gynecology Scenario:}

A 32 year old woman presented to the emergency department complaining of heavy vaginal bleeding with fever. She gave a history of a recent genital infection for which she was given tinidazole. Her Temperature is 39 C, blood pressure $80 / 50 \mathrm{mmHg}$, the pulse 110 and respiration 18. Her abdomen is, tender and mildly distended. Her pelvic examination reveals approximately 
$200 \mathrm{ml}$ of clotted blood in the vagina, an open cervical os with tissue protruding from it associated with offensive mucopurulent discharge, and a 10 -week sized tender uterus. WBC $19000 / \mathrm{mm}$, Hct $22 \%$, platelets count $275,000 / \mathrm{mm}$, quantitative beta human chorionic gonadotropin is $100,000 \mathrm{mIU} / \mathrm{L}$. Lactic acid was increased at 6.2 (1.5-2.3). Pelvic ultrasound shows an echogenic material within the uterine cavity consistent with blood or tissue, no adnexal masses, and no free fluid. No viable pregnancy was seen.

* What is your bedside clinical diagnosis ( the diagnosis that would explain all the symptoms and signs ): Septic shock secondary to incomplete abortion

* What is your likeliest bedside etiologic etiopathological cause of above bedside clinical diagnosis: Genital tract infection with Trichomonas vaginalis

* What is your bedside severity score: severe

List at least 5 essential immediate therapeutic interventions:

- Site of care: Critical care

- Symptomatic intervention: Antipyretics

- Supportive intervention: intravenous fluids, blood transfusion.

- Specific intervention: Dilation and curettage, antibiotics

- Specialty referral: Intensivist, Gynecologist

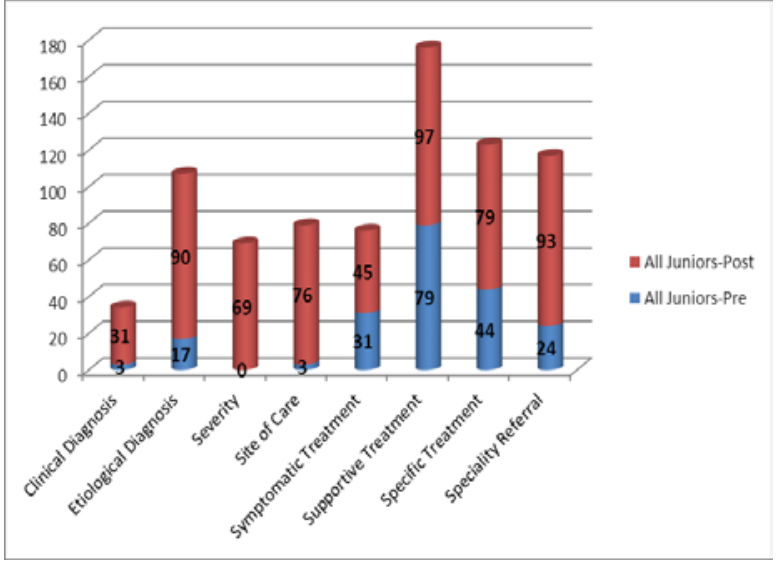

Figure 2: Changes in Care -Output following the use of Management Concept Maps in Juniors

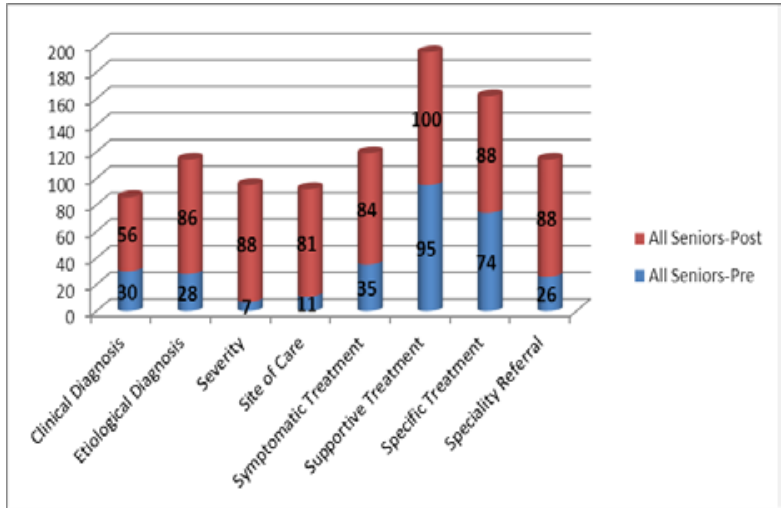

Figure 3: Changes in Care -Output following the use of Management Concept Maps in Seniors

Table 2: All Departments’ Trainees Pre-and Post-Results

\begin{tabular}{|c|c|c|c|c|c|c|c|c|}
\hline \multirow[t]{2}{*}{ Total Number } & \multicolumn{3}{|c|}{$N=29$} & \multirow[b]{2}{*}{ p-value } & \multicolumn{3}{|c|}{$N=43$} & \multirow[b]{2}{*}{ p-value } \\
\hline & $\begin{array}{l}\text { Juniors } \\
\text { Pre (\%) }\end{array}$ & $\begin{array}{c}\text { Juniors } \\
\text { Post (\%) }\end{array}$ & $\begin{array}{c}\text { Improvement } \\
(\%)\end{array}$ & & $\begin{array}{l}\text { Seniors } \\
\text { Pre (\%) }\end{array}$ & $\begin{array}{c}\text { Seniors } \\
\text { Post (\%) }\end{array}$ & $\begin{array}{c}\text { Improvement } \\
(\%)\end{array}$ & \\
\hline Clinical Diagnosis & 20.7 & 69 & 48.3 & $<0.005$ & 41.9 & 76.7 & 34.9 & $<0.005$ \\
\hline Etiological Diagnosis & 10.3 & 93.1 & 82.8 & $<0.005$ & 34.9 & 95.3 & 60.5 & $<0.005$ \\
\hline Severity & 3.4 & 82.8 & 79.3 & $<0.005$ & 4.7 & 93 & 88.4 & $<0.005$ \\
\hline Site of Care & 0 & 86.2 & 86.2 & $<0.005$ & 14 & 90.7 & 76.7 & $<0.005$ \\
\hline Symptomatic Treatment & 27.6 & 96.6 & 69 & $<0.005$ & 32.6 & 97.7 & 65.1 & $<0.005$ \\
\hline Supportive Treatment & 93.1 & 100 & 6.9 & 0.15 & 97.7 & 100 & 2.3 & 0.90 \\
\hline Specific Treatment & 55.2 & 89.7 & 34.5 & $<0.005$ & 62.8 & 83.7 & 20.9 & 0.028 \\
\hline Specialty Referral & 13.8 & 89.7 & 75.9 & $<0.005$ & 23.3 & 81.4 & 58.1 & $<0.005$ \\
\hline
\end{tabular}

\section{Discussion}

The Dreyfus brothers in the eighties, disseminated the five-stage development continuum that consists of novice, advanced beginner, competent, proficient, and expert. According to them and compared to a novice, an expert is quickly and fluently able to retrieve knowledge and articulate "the best course of action” [16]. In our exercise, the concept maps guided and helped trainees make diagnostic labeling more precise and comprehensive and the immediate therapeutic interventions more patient-focused incorporating important 
contextual factors for a more comprehensive, guideline-friendly, care in-put. The maps clearly assisted in realizing most of the institute of Medicine quality domains. An interesting observation in our brief exercise using the diagnostic labeling concept map is the difficulty trainees had (specially the juniors) in discriminating between the bed-side acute clinical diagnosis and the etiological diagnosis or actual precipitant of the acute condition-a vital differentiation for proper, focused and comprehensive care input. Similarly, trainees, almost consistently failed to include a severity score or assessment, an appropriate site of care and any need for specialty referral. On the whole, seniors scored better in all domains of both maps most likely because of their higher experience and knowledge.

In Medicine, clinical decision making is thought of as comprising two distinct yet intricately interrelated cognitive processes. The first mental exercise is related to the clinician's knowledge and skill in reaching a clinical bed-side diagnosis and in formulating a therapeutic plan. The second component has to primarily deal with the contextual aspects or features of the patient and his/her disease. The latter component guides the "good" clinician in putting together a patientfocused, individualized, evidence-based care plan. This comprehensive process of critical thinking and problem-solving so called "reflective judgment" is a hallmark of expert decision-making [17, 18]. It is generally believed that clinicians utilize two modes of reasoning or decision processes-as quoted by Sladek et al. [3] One (System1) has been described as "experiential, unconscious, fast, quick, intuitive, recognition primed, implicit, automatic and acquired via biology or exposure'[3]. The other mode (System 2) has been described as "rational, conscious, deliberate, slow, rule-based, analytic, explicit, controlled, and acquired by cultural and formal tuition" [3]. The remarkable improvement in diagnostic labeling and therapeutic care decisions (without any further educational input) probably proves that the "incomplete" initial care decisions were not due to a "lack of knowledge" but rather a cognitive "application of knowledge" deficit. Thus the maps allowed for a seamless contextual application of acquired knowledge. It probably does so by forcing the trainee to move from the fast, superficial and intuitive cognitive decision process (System 1) to the more robust, rational and analytic one (System 2) [3,19]; an achievement that may be more conducive to a better critical thinking, decision-making and the use of evidence-based, medical care. It is well-recognized that didactic educational interventions are associated with limited improvements in practice [20-22]. This is probably because didactic knowledge acquisition is not usually coupled with audiences' empowerment with practical conceptual maps and frameworks for knowledge application. As training or educational tools, our concept maps conform to several educational theories being adult-learner friendly (need, problem and experience-centered), applicable to the constructivist theoretical learning principle and simple to learn (low cognitive load) [4-6].

Cognitive conceptual deficiencies in junior staff decision making have been shown to be an important cause for poor outcome in the acute care setting (diagnosis, management decisions and delayed consultations) [23]. Our concept maps may thus also help reduce cognitive mistakes in the emergency room. Tools for knowledge translation and evidence-based medicine implementation such as electronic and manual reminders and decision support systems such as clinical pathways, protocols, order sets, checklists, use of computerized decision support tools, mechanisms for error detection and rectification are but few examples of systemwide interventions for reducing these cognitive deficits [24-27]. Other individual or learnerfocused interventions to improve critical thinking and decision-making include simulation training in decision-making, case discussions with senior and experienced staff and assignments and critique of patient-care [8, 28, 29]. One fundamental principle of the Continuous Quality Improvement process include "an emphasis on raising the general level of care rather than focusing on pockets of poor practice" [30]. The use of these maps by all staff in clinical decision making may be conducive to a general 
improvement in care in-puts and teamwork. Additionally, including and incorporating all the items in these maps in staff's paper-based or electronic reminders like Clinical Pathways and Order Sets may help further enrich these tools and make them more evidence-based.

The two main limitations of this study are its invitro nature and the biases inherent to a beforeafter study design. A prospective study following a full incorporation of these maps in trainees' teaching curricula may shed light on their longer term merit (in-vivo) in patient care and knowledge translation.

\section{Conclusion}

In summary, our two simple generic concept maps improved the "contextual conceptualization”, decision-making (critical thinking and problem-solving) and quality of care outputs for residents across all the major general disciplines of medicine, surgery, pediatrics and obstetrics and gynecology (invitro). This was achieved without any additional educational input confirming that the initial deficient out-put was not related to poor knowledge. We recommend including these models as routine concept maps in residents' training curricula and clinical training at the bedside, morning meetings etc. We also advocate including these model headings as an essential component of any integrated care clinical pathways, order-sets etc.

This article highlights three main messages that are:

a. Cognitive concept maps are useful decision aids especially for front-line clinicians and care-givers.

b. Routine use of these two generic concept maps (BESD and 5S) may improve the use of analytic System 2 mode of thinking.

c. We recommend including these concept maps in clinical training curricula and in the trainees daily clinical activities e.g. acute case discussions in the morning meetings etc.

\section{Contributorship/ interests:

Dr Hassan has developed the concept maps based on his experience in clinical training, evidence-based medicine and knowledge translation. All the other authors have contributed to study design, concepts testing, analysis and writing the manuscript.

No funding was received and the authors declare that they have no competing interests or conflict of interest

\section{Reference}

1. Committee on the Quality of Health Care in America. Crossing the quality chasm: A new health system for the 21st century. Washington, DC: National Academy Press; 2001.

2. Grimshaw JM, Eccles MP, et al. Knowledge translation of research findings. Implement Sci. 2012;7(1):50.

3. Sladek RM, Phillips PA, Bond MJ. Implementation science: $\quad \mathrm{a}$ role for parallel dual processing models of reasoning? Implement Sci. 2006;1:12.

4. Mann KV. Theoretical perspectives in medical education: past experience and future possibilities. Med Educ. 2011;45(1):60-8.

5. Schumacher DJ, Englander R, Carraccio C. Developing the master learner: applying learning theory to the learner, the teacher, and the learning environment. Acad Med. 2013;88(11):1635-45.

6. Knowles M, Holton EI, Swanson R. 2005. The adult learner: the definitive classic in adult education and human resource development. Burlington, MA: Elsevier.

7. Mandin H, Jones A, Woloschuk W, Harasym P. Helping students to think like experts when solving clinical problems. Acad Med. 1997;72:173-9.

8. Hendricson WD, Andrieu SC, Chadwick DG, et al. ADEA Commission on Change and Innovation in Dental Education. Educational strategies associated with devel opment of problem-solving, critical thinking, 
and self-directed learning. J Dent Educ. 2006;70(9):925-36.

9. Novak JD, Gowin DB. Learning How to Learn. New York, NY: Cambridge University Press 1984.

10. Daley BJ, Torre DM. Concept maps in medical education: an analytical literature review. Med Educ. 2010;44(5):440-8.

11. Anderson LA, Day KL, Vandenberg AE. Using a concept map as a tool for strategic planning: The Healthy Brain Initiative. Prev Chronic Dis. 2011;8(5):A117.

12. Hassan I. Models for Enhancing Competency-Based Training and Contextual Clinical Decision Making. Clin Teach. 2012;9(6):392-7. .

13. Global Initiative for Asthma. GINA workshop report: global strategy for asthma management and prevention. Available at: http://www.ginasthma.com/.

14. Kitabchi, AE, Umpierrez, GE, Miles, et al. Hyperglycemic crises in adult patients with diabetes: a consensus statement from the American Diabetes Association. Diabetes Care 2009; 32:1335-43.

15. Motov SM, Khan AN. Problems and barriers of pain management in the emergency department: Are we ever going to get better? J Pain Res. 2008;2:5-11.

16. Dreyfus HL, Dreyfus SE. Mind over machine: the power of human intuition and expertise in the era of the computer. New York: Free Press, 1986.

17. Bordage G, Lemieux M. Semantic structures and diagnostic thinking of novices and experts. Acad Med 1991;66:S70-S72.

18. Chapman GB, Sonnenberg F, eds. Decision making in health care: theory, psychology, and applications. New York: Cambridge University Press, 2000.

19. Markovits H, Forgues HL, Brunet ML. More evidence for adual-process model of conditional reasoning. Mem Cognit. 2012;40(5):736-47.

20. WP, Sibley JC, Neufeld VR, Sackett DL, Gerrard BA. The impact of continuing education on the quality of patient care. Rudnick KV, Fraser Can Fam Physician. 1983;29:810-8.
21. Sibley JC, Sackett DL, Neufeld V, et al. A randomized trial of continuing medical education. N Engl J Med. 1982;306(9):5115.

22. Forsetlund L, Bjørndal A, Rashidian A, et al. Continuing education meetings and workshops: effects on professional practice and health care outcomes. Cochrane Database Syst Rev. 2009;(2):CD003030.

23. Farnan JM, Johnson JK, Meltzer DO, et al. Resident uncertainty in clinical decision making and impact on patient care: a qualitative study. Qual Saf Health Care. 2008;17(2):122-6.

24. Bowen JL. Educational strategies to promote clinical diagnostic reasoning. N Engl J Med. 2006;355:2217-25.

25. Croskerry P. The importance of cognitive errors in diagnosis and strategies to minimize them. Acad Med. 2003;78:775-80.

26. Ely JW, Graber ML, Croskerry P. Checklists to reduce diagnostic errors. Acad Med. 2011;86(3):307-13.

27. Hunt DL, Haynes RB, Hanna SE, Smith K. Effects of computer-based clinical decision support systems on physician performance and patient outcomes: a systematic review. JAMA. 1998;280:1339-46.

28. Bressan F, Buti G, Boncinelli S. Medical simulation in anesthesiology training. Minerva Anestesiol. 2007;73(12):1-11.

29. Peckler B, Schocken D, Paula R. Simulation in a high stakes clinical performance exam. J Emerg Trauma Shock. 2009;2(2):85-8.

30. Roger S. Continuous quality improvement: effects on professional practice and patient outcomes (Protocol for a Cochrane Review) Chichester, UK: John Wiley \& Sons, Ltd; 2004. 